\title{
Uncertainty and Sensitivity Studies with TRACE-SUSA and TRACE-DAKOTA by Means of Transient BFBT Data
}

\author{
Wadim Jaeger, ${ }^{1}$ Victor Hugo Sánchez Espinoza, ${ }^{1}$ \\ Francisco Javier Montero Mayorga, ${ }^{2}$ and Cesar Queral ${ }^{2}$ \\ ${ }^{1}$ Karlsruhe Institute of Technology, Institute for Neutron Physics and Reactor Technology, Hermann-von-Helmholtz-Platz 1, \\ 76344 Eggenstein-Leopoldshafen, Germany \\ ${ }^{2}$ Departamento de Sistemas Energeticos ETSI Minas, Universidad Politecnica de Madrid, Alenza 4, 28003 Madrid, Spain
}

Correspondence should be addressed to Wadim Jaeger; wadim.jaeger@kit.edu

Received 16 October 2012; Accepted 25 December 2012

Academic Editor: Borut Mavko

Copyright (C) 2013 Wadim Jaeger et al. This is an open access article distributed under the Creative Commons Attribution License, which permits unrestricted use, distribution, and reproduction in any medium, provided the original work is properly cited.

\begin{abstract}
In the present paper, an uncertainty and sensitivity study is performed for transient void fraction and pressure drop measurements. Two transients have been selected from the NUPEC BFBT database. The first one is a turbine trip without bypass and the second one is a trip of a recirculation pump. TRACE (version 5.0 patch 2) is used for the thermohydraulic study and SUSA and DAKOTA are used for the quantification of the model uncertainties and the evaluation of the sensitivities. As uncertain parameters geometrical values, hydraulic diameter, and wall roughness are considered while mass flow rate, power, pressure, and inlet subcooling (inlet temperature) are chosen as boundary and input conditions. Since these parameters change with time, it is expected that the importance of them on pressure drop and void fraction will change, too. The results show that the pressure drop is mostly sensitive to geometrical variations like the hydraulic diameter and the form loss coefficient of the spacer grid. For low void fractions, the parameter of the highest importance is the inlet temperature/subcooling while at higher void fraction the power is also of importance.
\end{abstract}

\section{Introduction}

Uncertainty and sensitivity studies are of increasing importance in the last couple of years since the foremost conservative codes have been updated and improved making them the so-called best-estimate codes. The licensing policy of nuclear installations in different countries requires now the quantification of uncertainties of the used physical models but also of input and boundary conditions of the bestestimate codes are applied. The combination of TRACE [1] and SUSA [2] and TRACE and DAKOTA [3] has proofed that the chosen tools are able to be used for such kind of tasks and an application to transient conditions is justified.

In the frame of code validation and verification it is mandatory to proof that the chosen code is able to represent stationary as well as transient behavior of nuclear power plants. The chosen transients are representative for typical
BWR transients involving the interaction of multiple systems. The first one is a turbine trip without bypass and the second one is a recirculation pump trip. In both transients, the power, the mass flow rate, and the outlet pressure have been varied as it would be expected during a real transient. The variation of these input and boundary conditions will cause a nonsteady behavior of the void fraction and pressure (drop) in the test bundle.

\section{BFBT Benchmark}

In a collaborate effort the OECD/NEA launched an international benchmark aimed to provide realistic BWR fuel assembly data for the validation and improvement of deterministic thermal hydraulic analysis codes. The benchmark [4] is divided into two phases consisting of different exercises. 
(i) Phase I: void distribution benchmark.

(a) Exercise 1: steady-state subchannel grade benchmark, where subchannel, meso- and microscopic approaches can be used.

(b) Exercise 2: steady-state microscopic grade benchmark, where meso- and microscopic approaches and molecular dynamics can be utilized.

(c) Exercise 3: transient macroscopic grade benchmark, where a subchannel approach can be applied.

(d) Exercise 4: uncertainty analysis of the void distribution benchmark.

(ii) Phase II: critical power benchmark.

(a) Exercise 0: steady-state pressure drop benchmark.

(b) Exercise 1: steady-state benchmark, which applies a one-dimensional approach with BT correlations and a subchannel mechanistic approach.

(c) Exercise 2: transient benchmark, which applies a one-dimensional approach with BT correlations and a subchannel mechanistic approach.

The focus of the present analysis is exercise 3 of phase I; transient macroscopic grade benchmark. Four transient scenarios have been investigated according to the specifications, a turbine trip without bypass, a recirculation pump trip, a recirculation pump stick, and a malfunction of the pressure control system. The first two transients are selected for the present study. The main geometrical features of the fuel assembly type C2A are given in Table 1.

\section{Modeling}

3.1. Brief Descriptions of the Codes. TRACE is used for the thermal hydraulic analysis of the BFBT fuel assembly. In the frame of a 2-Fluid, 6-Equation model the conservation equations for mass, energy, and momentum are solved for the liquid and vapor phase of water. By means of empirical models closure laws are provided in order to close the field equations. SUSA and DAKOTA are tools for uncertainty and sensitivity analysis. Based on information regarding the uncertainty parameters (e.g., reference value, range, and distribution) both codes use Monte Carlo based random sampling or Latin hypercube sampling methods in order to obtain a desired amount of parameter combinations. These combinations are equivalent to the number of inputs which have to be executed. The results will be evaluated. The change of the input parameters will be compared with the changes of the output parameters and by means of statistical measures a quantitative statement can be made about the importance or influence of an input parameter variation on the output.
TABLE 1: Main features of assembly C2A.

\begin{tabular}{lc}
\hline Parameter [unit] & Value \\
\hline Number of fuel rods [-] & 60 \\
Outer diameter [mm] & 12.3 \\
Rod pitch [mm] & 16.2 \\
Heated length [mm] & 3708 \\
Number of water rods [-] & 1 \\
Channel box inner width [mm] & 132.5 \\
Channel box corner radius [mm] & 8 \\
In channel flow area [mm $\left.{ }^{2}\right]$ & 9463 \\
Number of spacers [-] & 7 \\
Spacer type [-] & Ferrule \\
Spacer form loss coefficient [-] & 1.2 \\
Spacer location [mm] & 455, 967, 1479, 1991, 2503, \\
Heater outer diameter [mm] & 3015,3527 \\
Heater material [-] & 7.3 \\
Insulator outer diameter [mm] & Nichrome \\
Insulator material [-] & 9.7 \\
Cladding thickness [mm] & Boron nitride \\
Cladding material [-] & 1.3 \\
Axial power profile [-] & $600 /$ beryllium \\
\hline
\end{tabular}

3.2. Uncertain Parameters. The uncertain parameters for the transient analysis are given in Table 2 along with the uncertainty range and the distribution type [5]. For the temperature absolute values are reported. The inlet temperature varies by $\pm 1.5 \mathrm{~K}$. For the steady-state cases, the mean value is the actual temperature, for example, $500 \mathrm{~K}$ with a minimum of $498.5 \mathrm{~K}$ and a maximum of 501.5. This can be done since the temperature (single phase pressure drop) or inlet subcooling (for two-phase pressure drop and void fraction) is constant. But during the transients, the temperature is subject to change and therefore it is better if it ranges between -1.5 and $+1.5 \mathrm{~K}$. In case the inlet subcooling is expressed in terms of enthalpy it has been transformed into an inlet temperature.

During preliminary investigations, it turned out that DAKOTA (integrated in SNAP) is not accepting a mean value of 0.0 if the uncertainty range is added to the reference value $(500 \mathrm{~K}+X)$. Instead a multiplication factor has been chosen $(500 \mathrm{~K} \cdot X)$. That multiplication factor is $0.997-1.003$ (meaning that a temperature of $500 \mathrm{~K}$ will be multiplied with a factor which varies between 0.997 and 1.003).

3.3. Time Dependent Input and Boundary Conditions. The input and boundary conditions are given in Figure 1 for the inlet temperature (top left side), the bundle mass flow rate (top right side), the outlet pressure (bottom left side), and the assembly power (bottom right side).

The first 10 seconds are at steady-state conditions. For the turbine trip, at 10 seconds the turbine isolation valve 

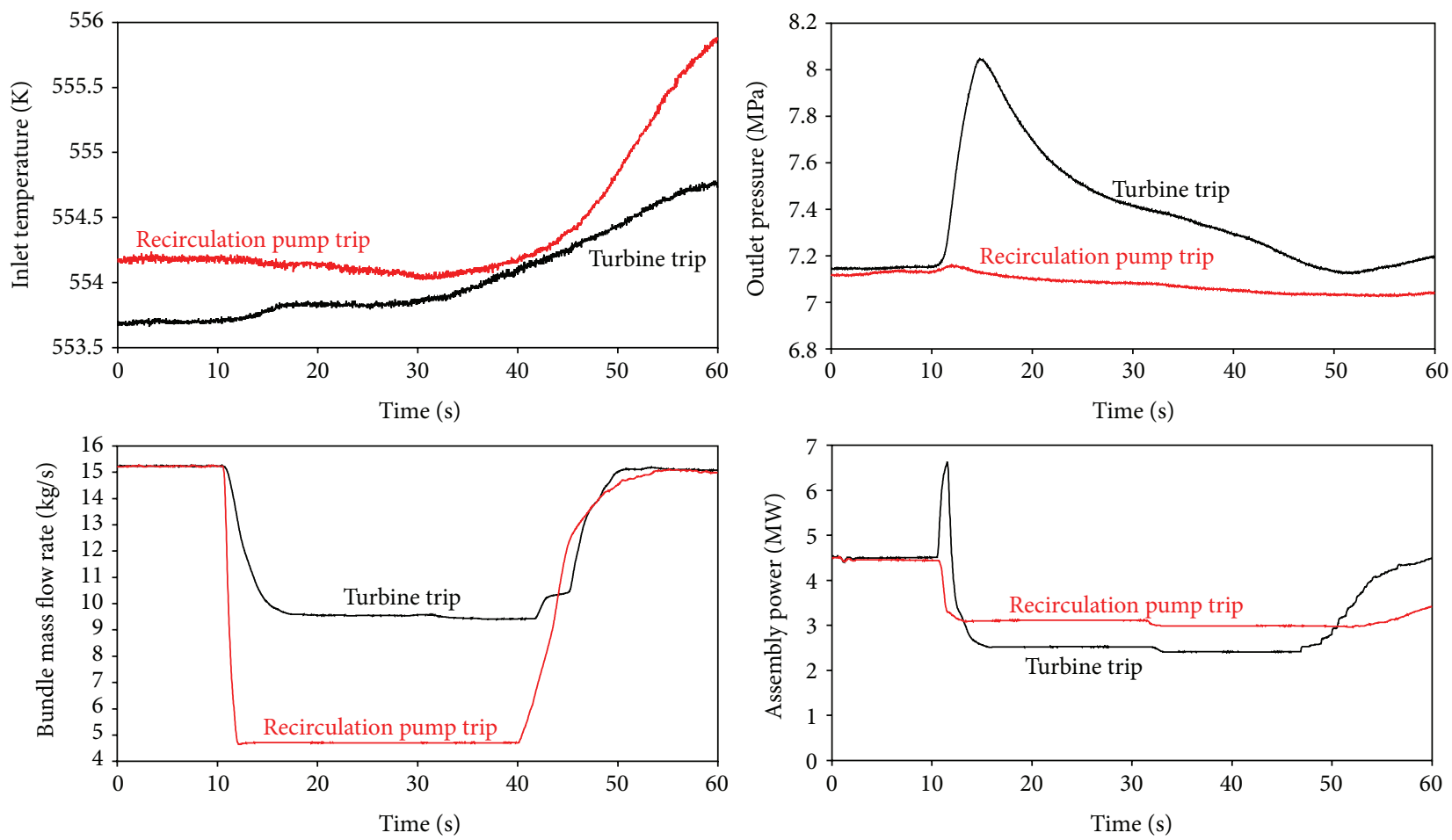

FIGURE 1: Input and boundary conditions during the transients.

TABLE 2: Uncertain parameters.

\begin{tabular}{lclcc}
\hline No. (SUSA/DAKOTA) & Parameter & Range & Distribution \\
\hline 1 & 1 & Outlet pressure & $\pm 1.0 \%$ & Normal \\
2 & 2 & Mass flow rate & $\pm 1.0 \%$ & Normal \\
3 & 3 & Inlet temperature & $\pm 1.5 \mathrm{~K}$ & Uniform \\
4 & 4 & Wall roughness & $\pm 5.0 \%$ & Normal \\
5 & 5 & K-spacer & $\pm 5.0 \%$ & Normal \\
6 & - & Hydraulic diameter & $\pm 1.0 \%$ & Normal \\
7 & - & Flow area & $\pm 1.0 \%$ & Normal \\
8 & 6 & Power & $\pm 1.5 \%$ & Normal \\
\hline
\end{tabular}

is closed causing a pressure wave backwards through the core. Therefore, the pressure in the system is increased which moves the saturation line to higher temperatures triggering the collapse of the void. In a nuclear power plant with feedbacks between thermal hydraulics and neutron physics, the increased density provokes a rise in the power due to the better moderation of the neutrons and the subsequent increasing fission rate.

In the experiment the assembly power has been increased to reasonable values to simulate these effects. As a consequence of the increased power, the mass flow rate is reduced being the preferred way of performing a power change.

In case of the turbine trip the mass flow rate drops to about $66 \%$ of the nominal value and is kept constant for the next 40 seconds. The pressure in the system is reduced till it reaches a value which is comparable to the initial one while the void fraction increases slowly due to the reduced saturation line. That happens after approximately 50 seconds transient time. The mass flow rate is increased to nominal values and the power stabilizes at values close to the ones of the nominal state.

For the recirculation pump trip, the mass flow rate is reduced after 10 seconds from $100 \%$ to around $30 \%$. With a lower mass flow rate more vapor is generated and the void fraction rises. The reduced density has a negative influence on the moderation and the power is decreasing. After about 40 seconds transient time, the normal mass flow rate is reestablished and the power rises consequently. In both transients the inlet temperature changes are only marginal but cannot be neglected since the distance to saturation temperature is reduced.

3.4. TRACE Model of the BFBT Fuel Assembly Mock-Up. The assembly type C2A [4], given in Figure 2, is modeled with the CHAN component in TRACE. The right side shows the axial nodalization of that CHAN. The model is sub-divided into 25 axial cells. At the bottom of the left side of that figure the FILL component (no. 100) is shown which is used to define the mass flow rate and inlet temperature boundary condition. A BREAK component (n. 300) is used for the pressure boundary condition. The lower left side shows the pin array with in total 25 pins as represented by the CHAN component. Five types of pins have been defined. Pins 1 till 4 represent regular heated rods and pin type five represents the water rod. Four water rods are present since the real water rod is taking the place of four regular rods. 

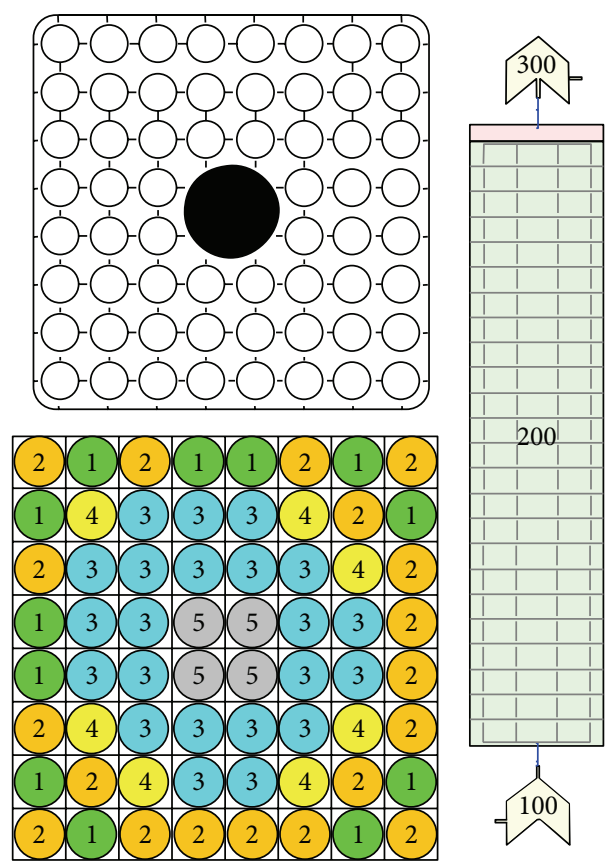

FIGURE 2: Sketch of assembly type C2A (top left), radial cross section (bottom left), and Axial nodalization (right side) of the TRACE model.

\section{Results}

For this investigation, 93 runs have been performed for the uncertainty and sensitivity analysis according to the specifications given in Section 3. Concerning the uncertainty analysis, statistical parameters like the minimum, maximum, and the mean are calculated based on the 93 runs. The minimum and maximum are evaluated at every time step; these are not the runs which yield the highest or smallest value.

Regarding the sensitivity analysis, Pearson's product momentum correlation coefficient is used as sensitivity coefficient. It is a statistical measure to evaluate the linear dependence between two variables (input on output parameter) and is defined as follows:

$$
r=\frac{\sum_{i=1}^{N}\left(x_{i}-\mu_{x}\right) \cdot\left(y_{i}-\mu_{y}\right)}{\left[\sum_{i=1}^{N}\left(x_{i}-\mu_{x}\right)^{2} \cdot \sum_{i=1}^{N}\left(y_{i}-\mu_{y}\right)^{2} \cdot\right]^{1 / 2}},
$$

where $x$ defines an input parameter (like the mass flow rate) and $y$ and output parameter (like the void fraction), $\mu$ is defined as the mean value (of e.g., all mass flow rates or void fractions) with a $x$ or $y$ as subscript to indicate the mean of the input or the output value. The subscript $i$ indicates the actual case (e.g., case 23) of the $N$ cases. In the present study $N$ is equal to 93 .
4.1. Pressure Drop. The measured and calculated pressure differences for the turbine trip and the recirculation pump trip are shown in Figures 3 and 4, respectively. The experimental data are characterized by a large spread of the measured data. The reason of that might be the used pressure sensor. The pressure during the steady state scenarios was measured with a differential pressure tap dpT9. But for the transients, measurements of the PTN010 and PTN007 are provided which are absolute pressure sensors. In the specification [4], an uncertainty of $1 \%$ is stated. A measured differential pressure of, for example, $10000 \mathrm{~Pa}$ would have a variation of $\pm 100 \mathrm{~Pa}$ which is marginal, but the absolute pressure sensors measure values of around 7-8 $\mathrm{MPa}$, as shown in Figure 1. $1 \%$ of $7 \mathrm{MPa}$ are 0.7 bar. If one takes now the difference of the inlet and the outlet, each one with an uncertainty of 0.7 bar, considering that the difference between inlet and outlet is less than 1 bar, the large spread is plausible.

Another point to address is that the TRACE predictions underestimate the measurements, between seconds $0-10$ and 45-60. Between second 10 and 40, TRACE is in agreement to the experimental data for the turbine trip but overestimates the conditions during the recirculation pump trip. The mean value of the experimental data for the first 10 seconds can be estimated to be 1.0-1.2 bar for both trips since the nominal mass flow rate, pressure, and power are similar/identical. The calculations are between 0.8 and 0.9 bar.

For the period where the mass flow rate has been reduced the experimental mean value is around 0.4 bar for the turbine trip and around 0.15 bar for the recirculation pump trip. Considering the mass flow rate reductions of the two scenarios, $100 \%$ to $66 \%$ for the turbine trip and $100 \%$ 


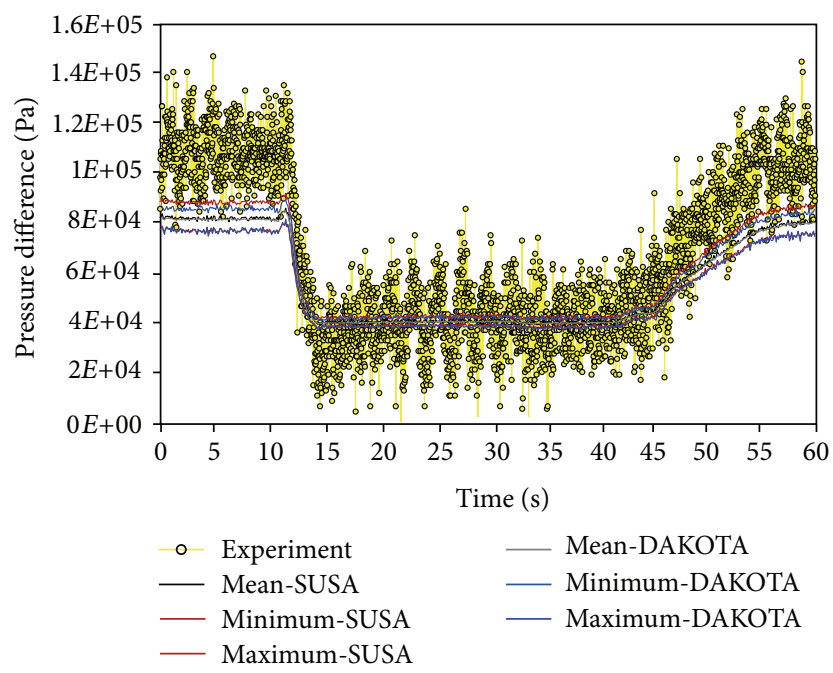

FIgURE 3: Pressure difference as a function of time for the turbine trip.

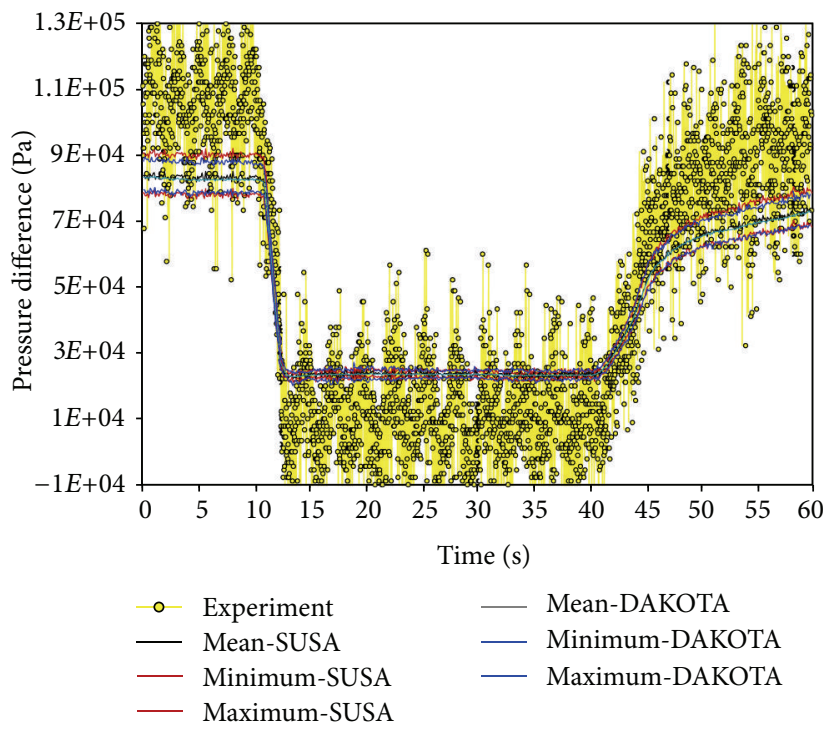

Figure 4: Pressure difference as function of time for the pump trip.

to $33 \%$ for the recirculation pump trip, the reduction of the experimental pressure drops is comprehensible. TRACE predicts similar values for the turbine trip, around 0.4 bar but slightly over estimates the 0.15 bar of the recirculation pump trip with 0.2 bar.

Besides the mass flow rate, the pressure and the power are changed during the transient. This will change the void fraction, see the next subsection, and subsequent the pressure difference. Therefore, it is not as trivial to determine to which extend the mass flow rate reduction is responsible for the reduced pressure drop. During a single phase flow a reduction of the mass flow rate of $50 \%$ will cause a reduction of the pressure drop to $25 \%$ which might not be applicable in the present case. Moreover, as explained, the calculated experimental pressure drop has a rather large uncertainty and as a consequence the presented values must be treated with caution.
The outcome of the uncertainty study is that at nominal condition the pressure drop is about $0.83 \pm 0.04$ bar while after the reduction the pressure drop is $0.39 \pm 0.02$ bar for the turbine trip and $0.235 \pm 0.01$ bar for the recirculation pump trip. That means that the error band is narrower with reduced mass flow rate. In addition, the error band of the TRACESUSA calculations envelops the one of TRACE-DAKOTA. The reason for that is the hydraulic diameter within the TRACE-SUSA investigation, which is not available for the TRACE-DAKOTA investigations

It also shows that the variation of the input and boundary conditions is not the reason why TRACE underestimates the first 10 seconds of the transient. The wrong prediction of the void fraction (the lower void fraction the higher the pressure drop) can be excluded as Section 4.2 will show. A reason why the differences are that considerably could be the physical 


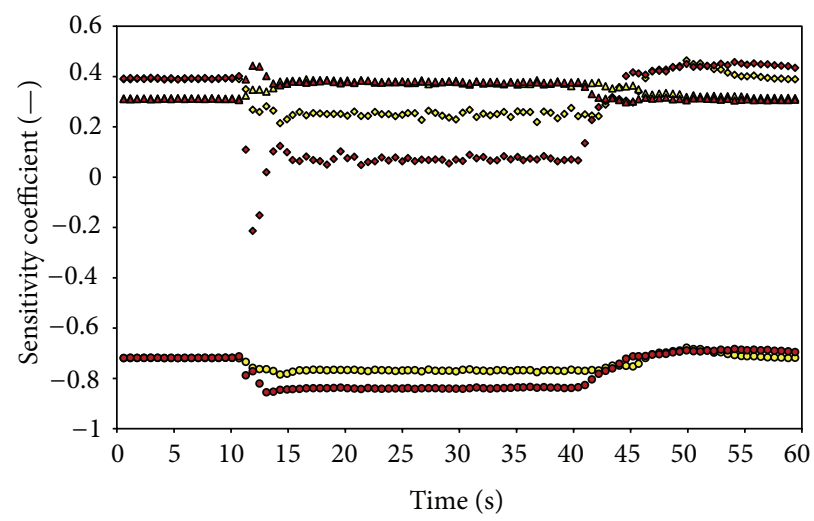

$$
\begin{aligned}
& \diamond \text { Inlet temperature (turbine trip) } \\
& \bigcirc \text { Hydraulic diameter (turbine trip) } \\
& \Delta \text { K spacer (turbine trip) } \\
& \diamond \text { Inlet temperature (pump trip) } \\
& - \text { Hydraulic diameter (pump trip) } \\
& \Delta \text { K spacer (pump trip) }
\end{aligned}
$$

FIGURE 5: Sensitivity coefficients related to the pressure drop for the turbine trip (yellow symbols) and the recirculation pump trip (red symbols) with TRACE-SUSA.

models used for calculating the two-phase flow pressure drop, but a comparative study with results obtained from subchannel and CFD codes shows that all codes underpredict the first 10 seconds. It could be also an indication that the form loss coefficient of the spacers is higher than the 1.2 which is considered here. That value has been mostly likely derived for single phase flow but is used also in two-phase flow. As Figures 3 and 4 indicate, the discrepancy is high at higher mass flow rate while the agreement is good at low mass flow rate. Since a fixed $\mathrm{K}$ factor is used, the mass flow rate (Reynolds number dependence) is not considered. In addition, the fact that the absolute pressure sensors have been used might also add some uncertainty.

Based on the uncertainty results it is interesting to see whether the importance of input parameter variations is changing in the same way as pressure drop does during the transient. The advantage of SUSA compared to DAKOTA (integrated in SNAP) is that also time dependent sensitivity coefficients can be calculated.

Such values are not available for DAKOTA. The sensitivity coefficients are plotted in Figure 5 for both transients. It can be seen that the parameter with the highest importance is the hydraulic diameter. For both transients similar even almost identical values are calculated. Only during the period with the reduced mass flow rate, the absolute value of the sensitivity coefficient is increased slightly where the increase during the pump trip is more pronounced. That means that with reduced pressure drop the importance of the hydraulic diameter is increased. The opposite behavior is found for the inlet temperature. During the reduced mass flow rate time period, the importance of these parameters is reduced. Especially for the inlet temperature the sensitivity coefficient changed from values close to 0.4 which indicates a medium correlation between it and the pressure drop to values less than 0.1 which indicates a rather weak dependence. The values for the form loss coefficient are around 0.3 and increase slightly with decreasing mass flow rate. It can be stated that for DAKOTA the sensitivity coefficients for the inlet temperature and form loss coefficient are much higher but following the same behavior as for SUSA.

4.2. Void Fraction. The transient void fractions are plotted for the three X-ray densitometer positions. Unfortunately, the $\mathrm{X}$-ray CT scanner information is only available for the first couple of seconds and has been therefore not considered in the graphical representation of the results. Figure 6 shows the comparison for the turbine trip while Figure 7 depicts the ones for the recirculation pump trip.

The presented experimental data are the corrected data. The correction was necessary due to the limitations of the X-ray densitometers, for more details refer to [6].

The changes of the input and boundary conditions are reflected in the void fraction trends. For the turbine trip one can see an increase followed by a sharp decrease. That is related to the power peak and the subsequent mass flow rate and power reduction and pressure peak. The void is increasing constantly after the pressure is decreased in an almost linear manner. Afterwards, the mass flow rate is increased which causes a reduction in the void. But shortly after, the power is increased too, which results in an increasing void.

It is also worth to mention that the calculated higher void fractions, for example, densitometer 3 at 40 seconds for the turbine trip, do not lead to dry out conditions.

The comparison of the experimental data with the mean values of TRACE-SUSA and TRACE-DAKOTA shows that TRACE is able to reproduce the experiment. Especially for the first two densitometer positions, the agreement is very good. For the third and highest position the deviations are visible. Nevertheless, the TRACE predictions are only around $10 \%$ higher than the experiment, for the period with reduced 

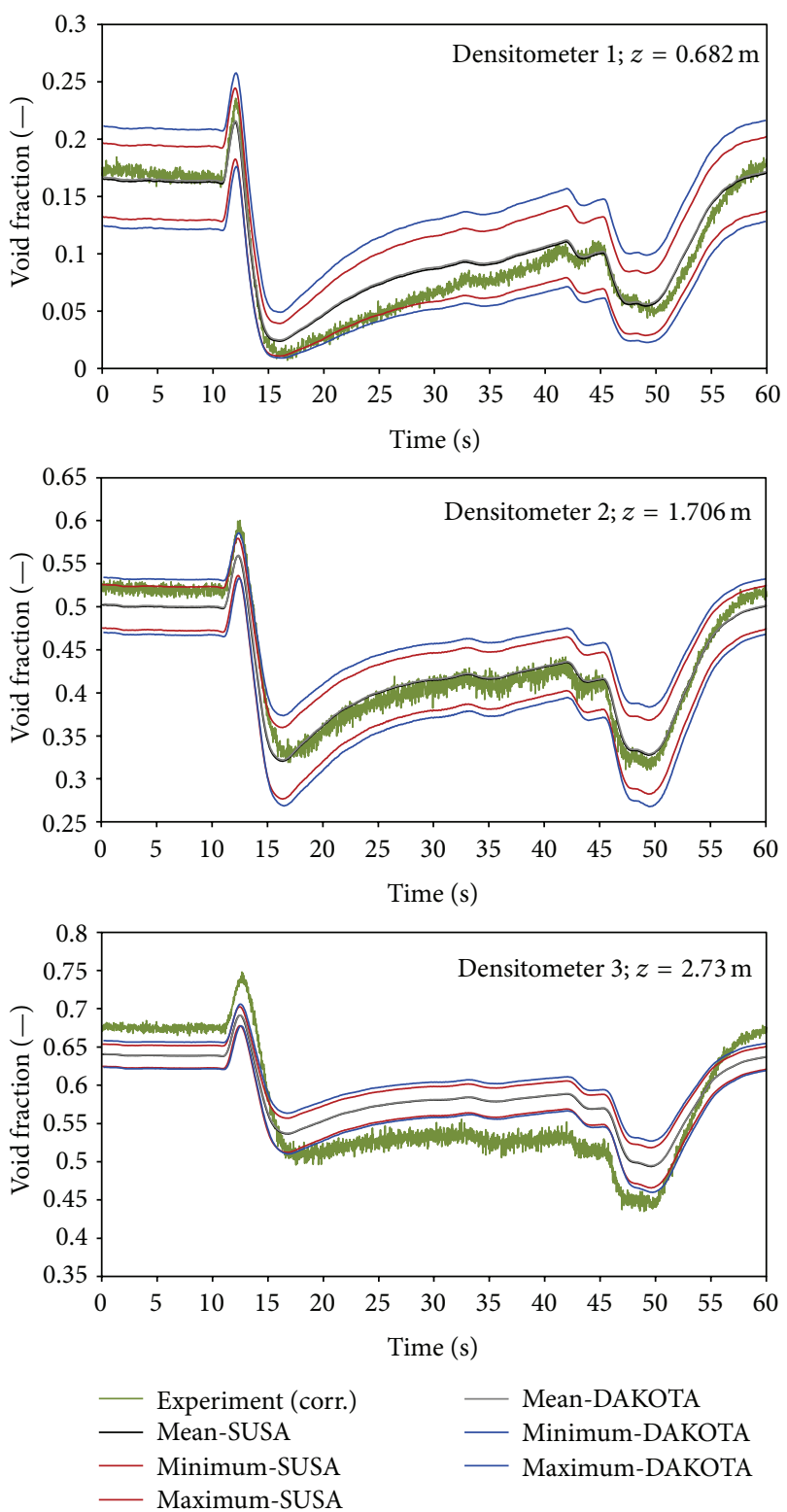

FIGURE 6: Transient void fraction during the turbine trip at different elevations.

mass flow rate. With increasing void fraction the uncertainty band is getting smaller. Even though the TRACE predictions are only $10 \%$ higher than the measurements, the uncertainty band is not enveloping them. The applied correction of the measurements followed the proposal of Glück [6]. That correction has been derived from experiments with different assembly types. Therefore, it cannot be guaranteed that the presented corrected experimental data are the real ones and uncertainty has to be considered. Furthermore, it must be kept in mind that the positions of the X-ray densitometers are not matching perfectly with the elevations at which the data have been calculated in the TRACE. As mentioned above, the heated length of the assembly $(3.708 \mathrm{~m})$ is subdivided into 25 cells.
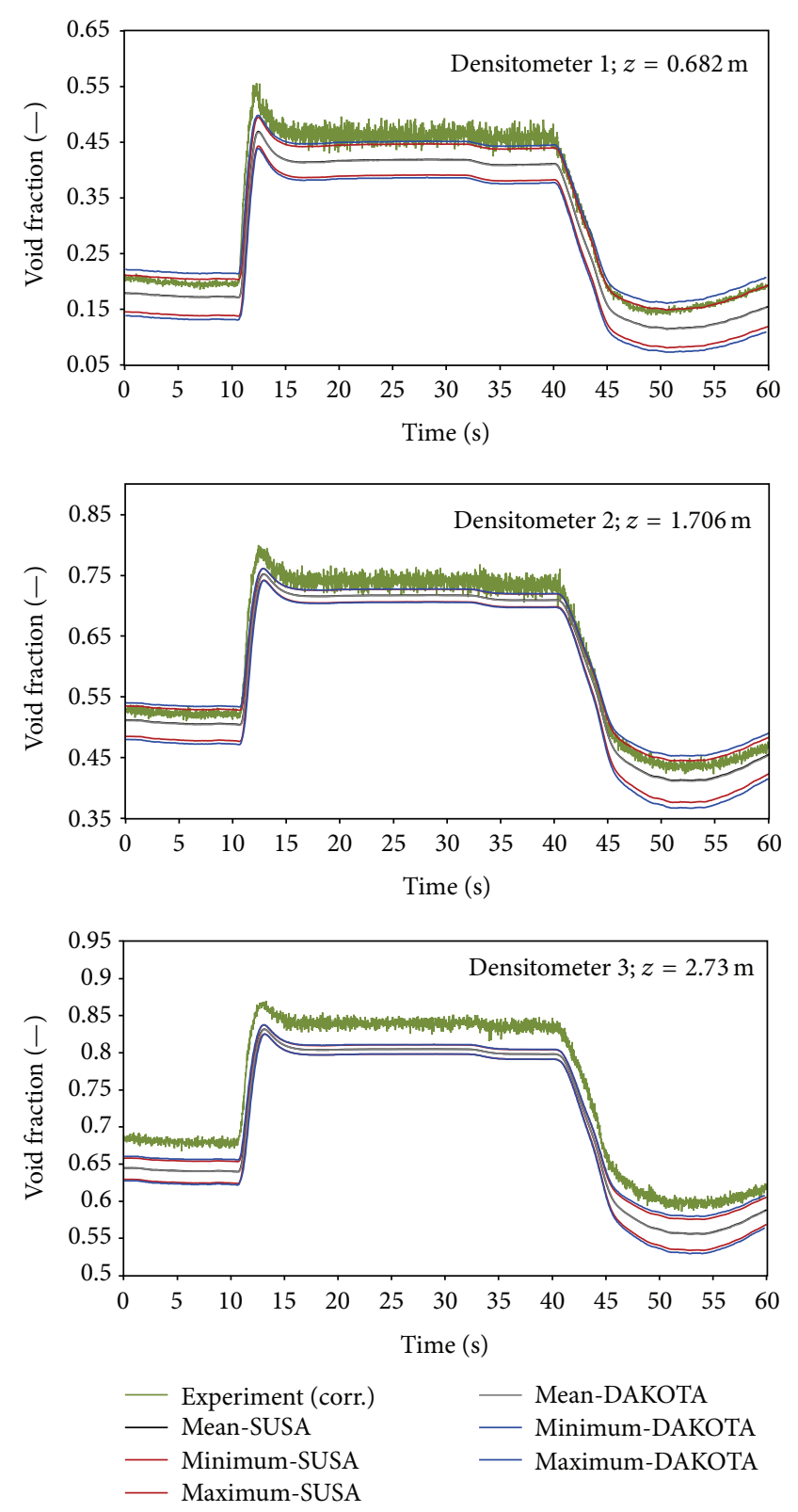

FIgURE 7: Transient void fraction during the pump trip at different elevations.

Regarding the recirculation pump trip, the void fraction trends are different from the one of the turbine trip. First of all, the reduction of the mass flow rate is more pronounced and second, the change of the power is less pronounced and no power peak is present either. In addition, the pressure stays constant during the trip. Therefore, the void fraction trends are in direct relation to the change of the mass flow rate and are nearly constant, between second 1 to 10,15 to 40 , and 45 to 60 seconds. As for the turbine trip, the TRACE results are in good agreement with the experiment. The average error for the three positions is in the order of $10 \%$ or lower. Only for the last $10-15$ seconds of the transient the deviations are more distinct. For that transient, a general underestimation of the 


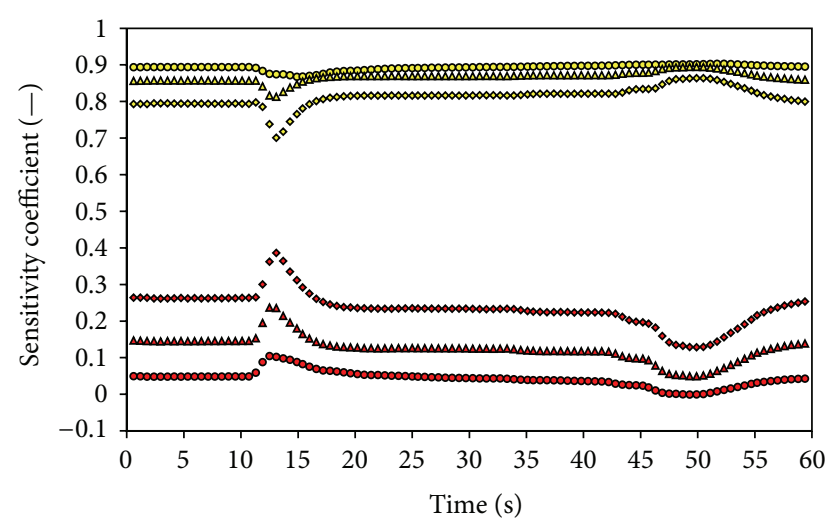

○ Inlet temperature (densitometer 1 )

$\triangle$ Inlet temperature (densitometer 2)

$\diamond$ Inlet temperature (densitometer 3)

- Power (densitometer 1)

$\triangle$ Power (densitometer 2)

$\diamond$ Power (densitometer 3 )

FIGURE 8: Sensitivity coefficients related to the void fraction during the turbine pump trip at three axial elevations (TRACE-SUSA).

experiment is visible. Also, the uncertainty band is smaller as for the turbine trip since the void fraction is in general higher.

One other important finding is that the uncertainty band of DAKOTA envelops the one of SUSA which should not be the case, especially since TRACE-SUSA employs one parameter more than TRACE-DAKOTA. Since the hydraulic diameter is not of importance in the present study for the formation of the void fraction, it is not responsible for that behavior. The reason is most likely the treatment of the inlet temperature. As mentioned previously, the $\pm 1.5 \mathrm{~K}$ uncertainty band is not considered. Instead the temperature at each time step is multiplied with a factor which ranges between 0.997 and 1.003. The differences between the two approaches are marginal since, for example, $554 \mathrm{~K}+1.5 \mathrm{~K}$ is 555.5 while $554 \mathrm{~K}$ times 1.003 is $555.62 \mathrm{~K}$, but the inlet temperature (inlet subcooling) is of importance for the void fraction evaluation and even small changes have a visible effect. This circumstance is confirmed by the sensitivity study. Figures 8 and 9 show the sensitivity coefficients for the turbine trip and the recirculation pump trip, respectively. In both plots the coefficients related to the inlet temperature and the power are shown for the three different axial positions which correspond to the X-ray densitometers.

First of all, the temperature is evaluated as the most important parameter at all. Values above 0.7 indicate a strong dependency upon the temperature. The power on the contrary, is of small or even no importance as values of less than 0.3 indicate. But with rising elevation, which corresponds to rising void fractions, the importance of the temperature is reduced while the power has a higher weighting. This behavior is more pronounced during the recirculation pump trip since the void fractions are generally higher than during the turbine trip. Especially after the mass flow rate reduction, the shifted weighting can be observed. For the densitometer 1 position the sensitivity coefficients for inlet temperature and

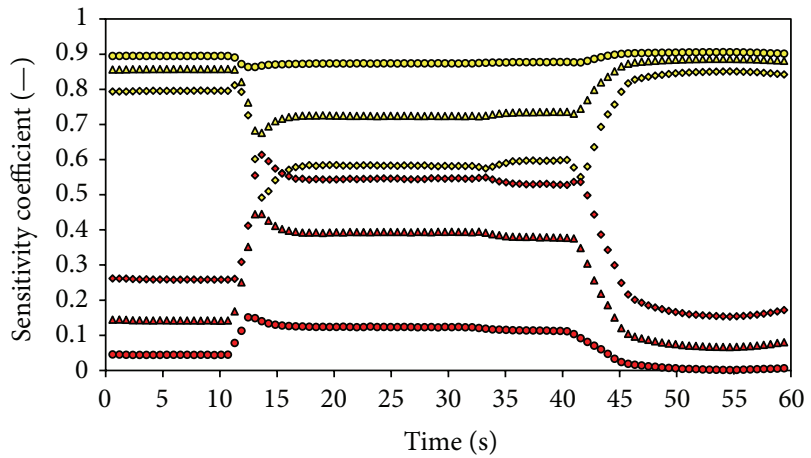

Inlet temperature (densitometer 1 )

$\triangle$ Inlet temperature (densitometer 2)

$\diamond$ Inlet temperature (densitometer 3)

- Power (densitometer 1)

$\triangle$ Power (densitometer 2)

$\diamond$ Power (densitometer 3)

FIGURE 9: Sensitivity coefficients related to the void fraction during the recirculation pump trip at three axial elevations (TRACESUSA).

power are almost constant. That can then also explain why the differences between the DAKOTA and SUSA uncertainty bands are more pronounced at lower elevations. For the recirculation pump trip, in particular at higher elevations, both bands are congruent.

\section{Summary and Conclusions}

TRACE simulations of a postulated turbine trip and a recirculation pump have been carried out in the frame of the presented investigation. The focus on this investigation was the quantification of the uncertainties of the pressure drop and the void fraction as a function of time. By means of input error propagation, the uncertainties related to input and boundary conditions as well as geometrical parameters provoked a variation of these parameters.

For both, the pressure drop and the void fraction, the comparison between experiment and TRACE prediction shows a very good agreement and a sound explanation is given for discrepancies. The performed uncertainty and sensitivity study with SUSA and DAKOTA shows in general a similar outcome. With respect to the pressure drop analysis, the variation of the hydraulic diameter has the largest portion on the uncertainty. Even though the hydraulic diameter is not included in the TRACE-DAKOTA analysis, the predicted uncertainty bands are similar though more tight. Concerning the void fraction, the sensitivity is related to the void fraction itself. At lower void fractions, the uncertainty band is mostly characterized by the change of the inlet subcooling, which has been considered by varying the inlet temperature. With increasing void fraction, the sensitivity coefficients for the inlet temperature are reduced while the power gains more and more importance.

Based on the results and the experience gained, it can be stated that with both code combinations reliable and sound results can be obtained. Nevertheless, the TRACE-SUSA 
tool is more flexible allowing a user and problem oriented treatment of the uncertain parameters and their uncertainties. Moreover, for future work, the possibility to include source code parameters is also of advantage. However, the usage of TRACE in conjunction with DAKOTA will become more frequently used since the implementation in a common graphical user interface (SNAP) allows a direct start while TRACE-SUSA requires programming skills. In the future, the capabilities of the integrated DAKOTA version will be extended.

One task for the near future is the incorporation of physical model parameters in the frame of uncertainty and sensitivity studies in order to evaluate their influence on the results. Unfortunately, no reliable data concerning uncertainty range and distribution of physical model parameters are available in a concentration that would allow a comprehensive study. The only thing which can be used up to now is a Phenomena Identification and Ranking Table (PIRT) which does not tell anything about the uncertainty but about the importance of parameters and models in general. Based on this information data must be provided for the selected physical models. In the frame of an ongoing OECD/NEA benchmark, international experts are working on the quantification of physical model parameters, which are sometimes eluding themselves from being measured. Based on the results of this benchmark, measures can be taken and the present transients can be recalculated.

\section{Conflict of Interests}

The authors hereby declare that no conflict of interests is present between them and the commercial entities mentioned in the context of the paper.

\section{Acknowledgments}

The authors thank the Program "Nuclear Safety Research" of KIT for the financial support of the Research Topic "Multiphysics Methodologies for Reactor Dynamics and Safety" and the Deutscher Akademischer Austausch Dienst (DAAD).

\section{References}

[1] U. S. NRC, TRACE V5. 0 Theory Manual-Field Equations, Solution Methods, and Physical Models, U.S. Nuclear Regulatory Commission, 2010.

[2] H. Glaeser, "GRS method for uncertainty and sensitivity evaluation of code results and applications," Science and Technology of Nuclear Installations, vol. 2008, Article ID 798901, 7 pages, 2008.

[3] B. Adams, K. Dalbey, M. Eldred, D. Gay, L. Swiler, W. Bohnhoff et al., DAKOTA, a Multilevel Parallel Object-Oriented Framework for Design Optimization, Parameter Estimation, Uncertainty Quantification, and Sensitivity Analysis Version 5.1 User's Manual, Sandia National Laboratories, Livermore, Calif, USA, 2011.

[4] B. Neykov, F. Aydogan, L. Hochreiter, K. Ivanov, H. Utsuno, F. Kasahara et al., NUPEC BWR Full-Size Fine Mesh Buncle
Test (BFBT) Benchmark Volume I: Specifications, OECD Nuclear Energy Agency, 2005.

[5] B. Neykov, L. Hochreiter, K. Ivanov, H. Utsuno, E. Sartori, and M. Martin, NUPEC BWR Full-Size Fine-Mesh Bundle Test (BFBT Benchmark) Volume II: Uncertainty and Sensitivity Analyses of Void Distribution and Critical Power-Specification, OECD Nuclear Energy Agency, 2007.

[6] M. Glück, "Validation of the sub-channel code F-COBRATF-part II: recalculation of void measurements," Nuclear Engineering and Design, vol. 238, no. 9, pp. 2317-2327, 2008. 


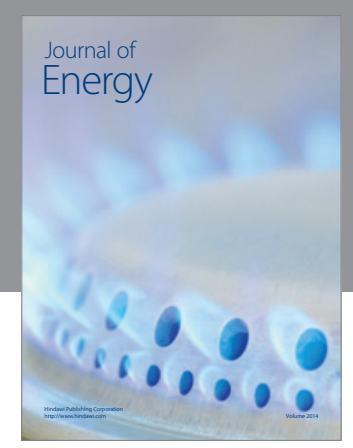

Journal of

Industrial Engineering
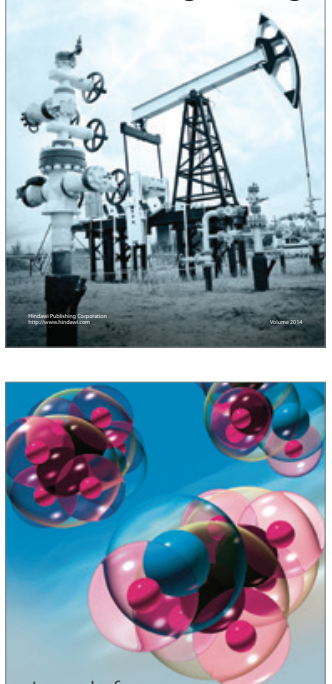

Fuels
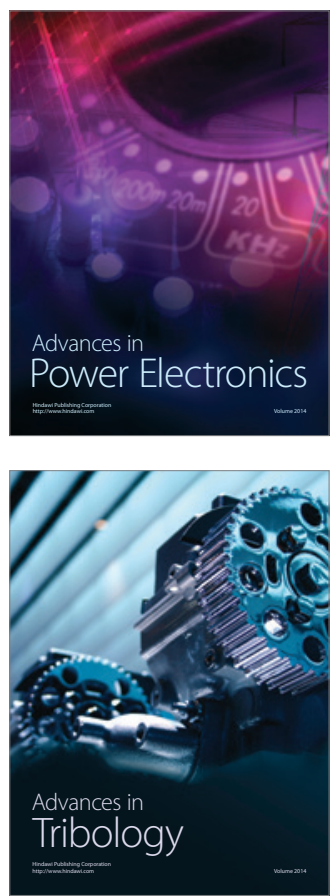

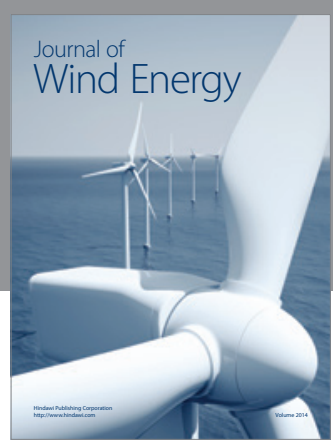

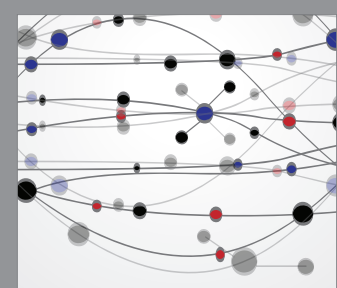

The Scientific World Journal

Submit your manuscripts at http://www.hindawi.com

Journal of

Structures
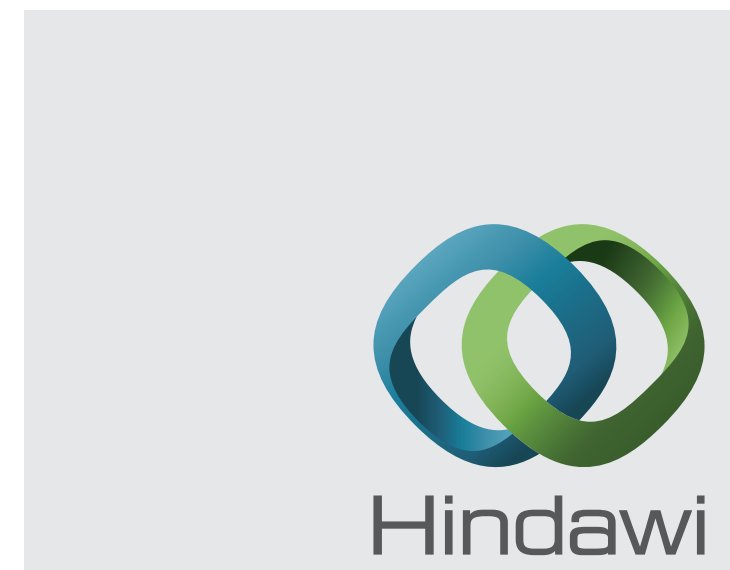

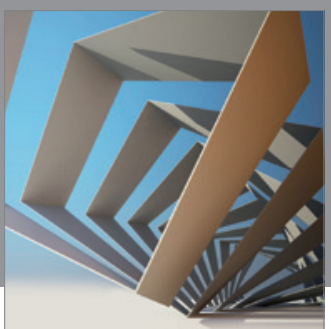

Rotating

Machinery
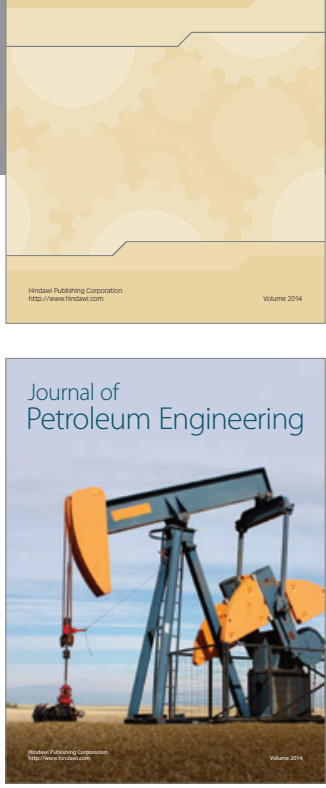

Journal of

Solar Energy
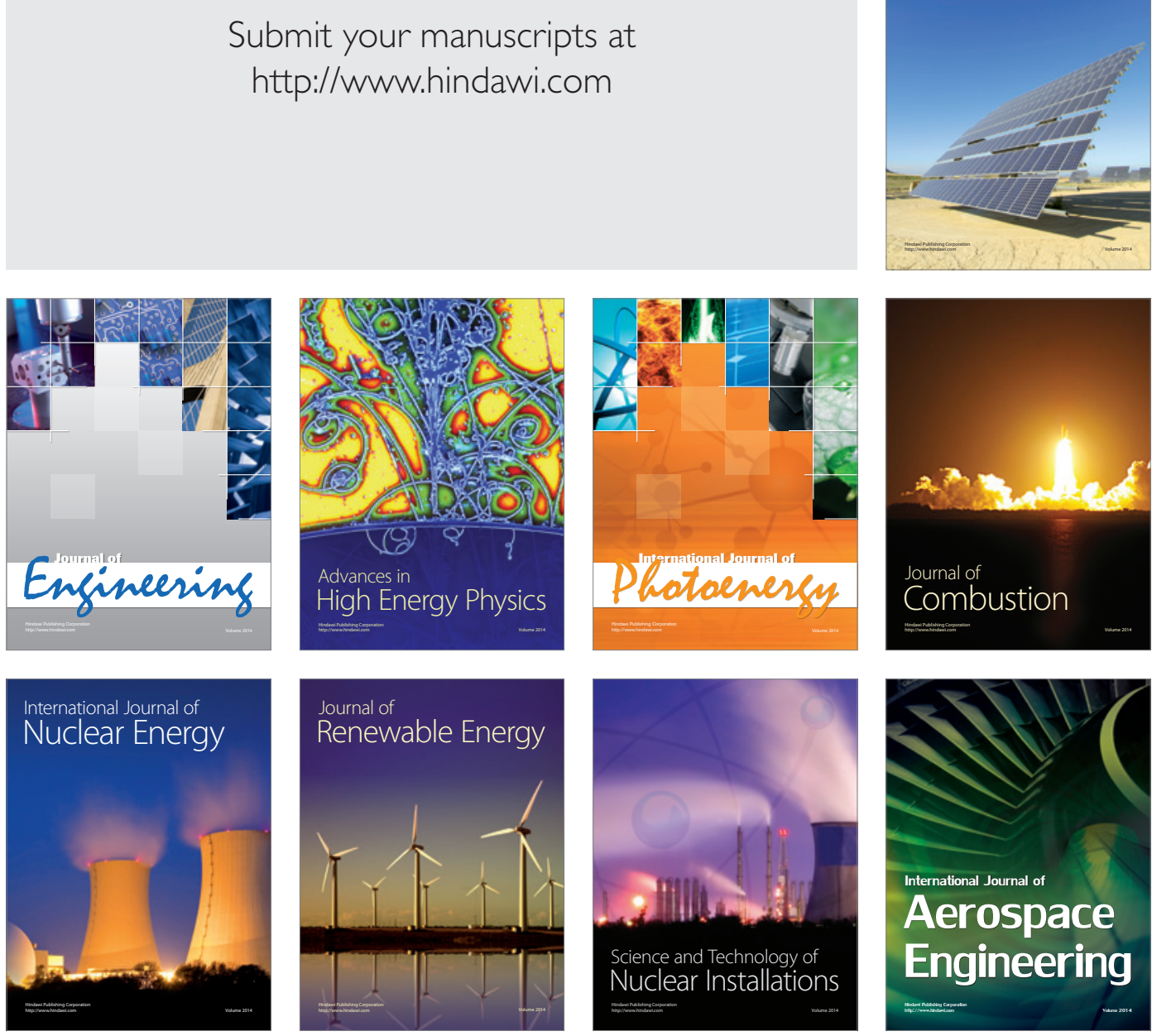